\title{
Quantitative Untersuchungen am Meiobenthos eines Profils unterschiedlicher Sedimente in der westlichen Ostsee*
}

\author{
W. SCHEIBEL \\ Zoologisches Institut der Universität Kiel; Kiel, Bundesrepublik Deutschland
}

\begin{abstract}
Quantitative investigations on the meiobenthos of a profile comprising various sediments in the western Baltic Sea. The investigations conducted provide a detailed picture of the colonization pattern of meiofauna on various sediments in the western Baltic Sea. Each sample core had an area of $10 \mathrm{~cm}^{2} \triangleq 50 \mathrm{~cm}^{3}$. Sampling was cartied out by hand with the help of divers. The animals found were removed with a pipette, counted, (copepods were identified) and weighed to ascertain their biomass. The average density of individuals $/ \mathrm{m}^{2}$ varies, according to the type of sediment, from 300,000 to $1,950,000$. Biomass was determined at 50 to $560 \mathrm{mg} / \mathrm{m}^{2}$ (dry weight). The sediment structure was demonstrated to be very important for the composition of meiofauna.
\end{abstract}

\section{EINLEITUNG}

Die vorliegenden Untersuchungen wurden im Versuchsgebiet Boknis Eck des Sonderforschungsbereiches 95 durchgeführt und sollen dazu beitragen, die in diesem Gebiet seit einiger Zeit laufenden physikalisch-chemischen, planktologischen und benthologischen Untersuchungen zu ergänzen. Im Bereich Boknis Eck besteht die Möglichkeit, mehr oder weniger alle in der Kieler Bucht vorkommenden Sediment-Typen auf engem Raum zu untersuchen. Die Konsistenz der Sedimente reicht von Kies bis zu Schlick.

Die bisherigen Arbeiten über Meiofauna im sublitoralen Bereich der Kieler Bucht hatten vor allem qualitativen Charakter (z. B. Ax, 1951, 1952; Remane, 1933; Kunz, 1935; Klie, 1929, 1950; Hartmann, 1956; Gerlach, 1958; Becker, 1970); quantitative Aspekte finden sich erst bei SCHeibel. (1973) sowie Scheibel \& NoodT (1975).

Die vorliegende Arbeit soll die bisher gewonnenen Daten über die Meiofauna auf unterschiedlichen Sedimenten abrunden. Es werden quantitative Proben über einen mehrmonatigen Zeitraum untersucht, die auf einem sublitoralen Profil entnommen wurden. Die Probennahme reichte von März 1973 bis Juni 1974. Für die Monate August, November und Dezember 1973 liegen aus technischen Gründen keine Proben vor. Deshalb wird im Rahmen dieser Arbeit nicht auf den Jahresgang eingegangen, sondern es werden nur die Durchschnittswerte behandelt.

$*$ Beitrag des Sonderforschungsbereiches 95, Nr. 86. 
Der Schwerpunkt der Arbeit liegt auf dem Besiedlungsmuster der Meiofauna. Außerdem wurde die Biomasse ermittelt. Detaillierter wird die Gruppe der Harpacticoiden (Copepoda) behandelt.

\section{MATERIAL UND METHODE}

Auf vier Tiefenstufen $(6-8 \mathrm{~m} ; 12-15 \mathrm{~m} ; 20-22 \mathrm{~m} ; 26 \mathrm{~m})$ wurden von März 1973 bis Juni 1974 (mit Ausnahme der Monate August, November und Dezember) einmal pro Monat quantitative Proben entnommen. Als Markierung dienten die Begrenzungstonnen des Sperrgebietes. Die Sedimentkerne wurden von Tauchern per Hand gestochen; jeder Kern umfaßt eine Fläche von $10 \mathrm{~cm}^{2} \triangleq 50 \mathrm{~cm}^{3}$. Zusätzlich wurde jeweils eine Parallelprobe gleicher Menge zur Bestimmung der Biomasse genommen. Die in 4 prozentigem Formalin fixierten Probenkerne wurden durch Siebe von 350 und $53 \mu$ gespült und die Meiofauna abpipettiert. Die Ermittlung der Biomasse erfolgte durch direktes Wägen als Trockengewicht. Die Proben wurden auf Filtern bei $60^{\circ} \mathrm{C}$ zwei Stunden lang im Trockenschrank getrocknet. Zur Verfügung stand eine Mettler-Feinwaage M5 SA.

\section{DAS UNTERSUCHUNGSGEBIET UND DIE VERTEILUNG DER SEDIMENTE}

Das Untersuchungsgebiet („Hausgarten“) des SFB 95 liegt im Westen der Kieler Bucht am Ausgang der Eckernförder Bucht (Abb. 1). Eingeschlossen wird ein Bereich,

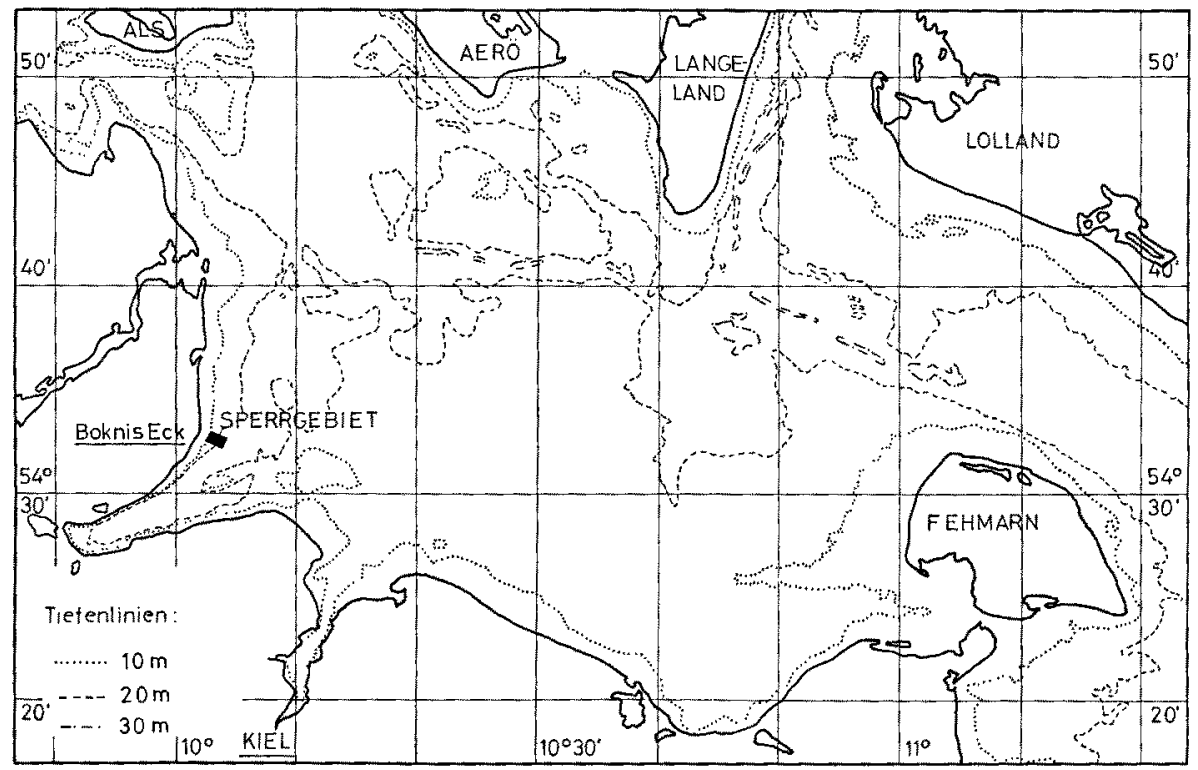

Abb. 1: Lage des Sperrgebietes des Sonderforschungsbereiches 95 in der Kieler Bucht 


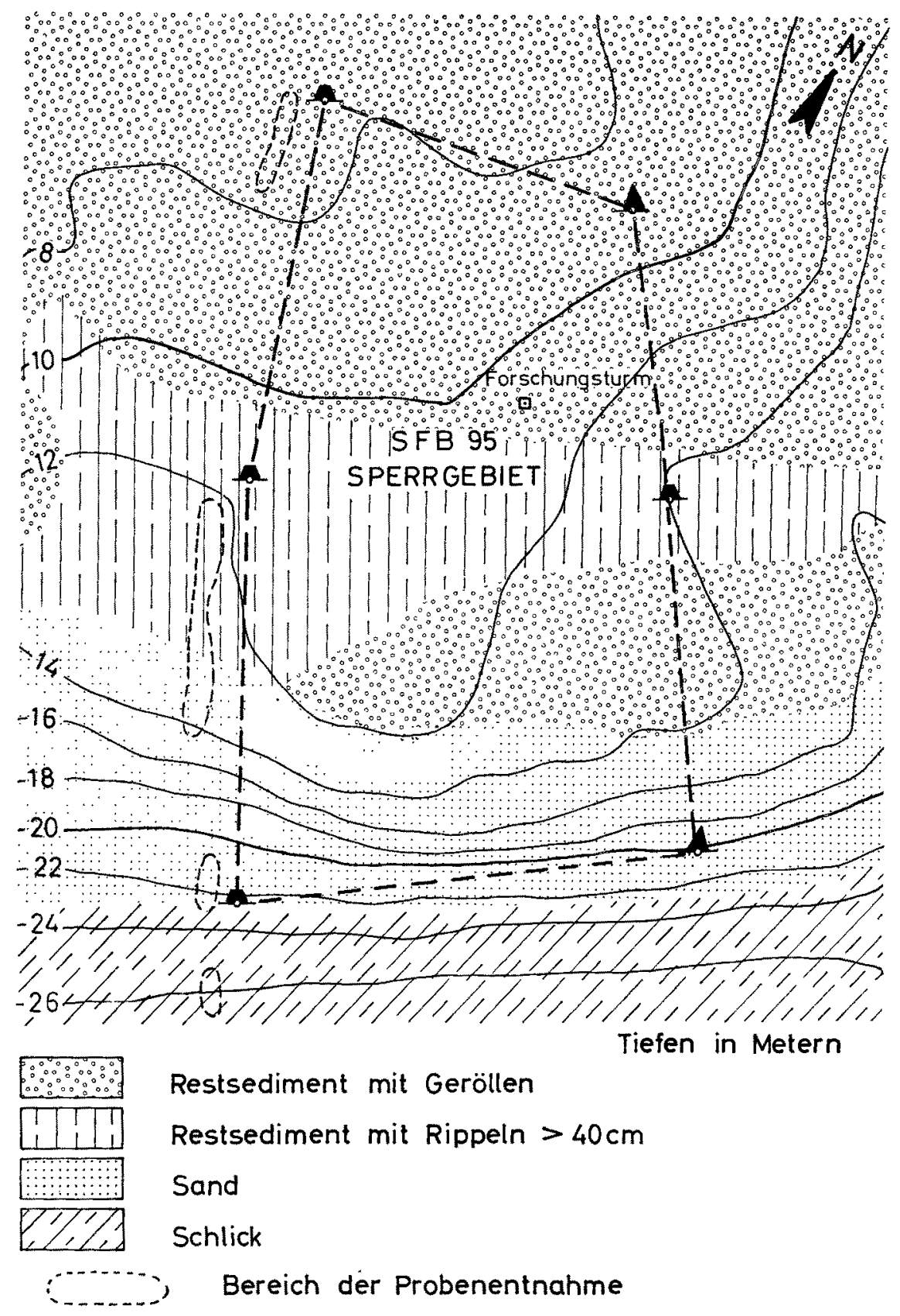

Abb. 2: Sedimentverteilung innerhalb des Untersuchungsgebietes des Sonderforschungsbereiches 95 im Ostseegebiet bei Bokniseck (nach WEFER und Tauchgruppe Kiel) 

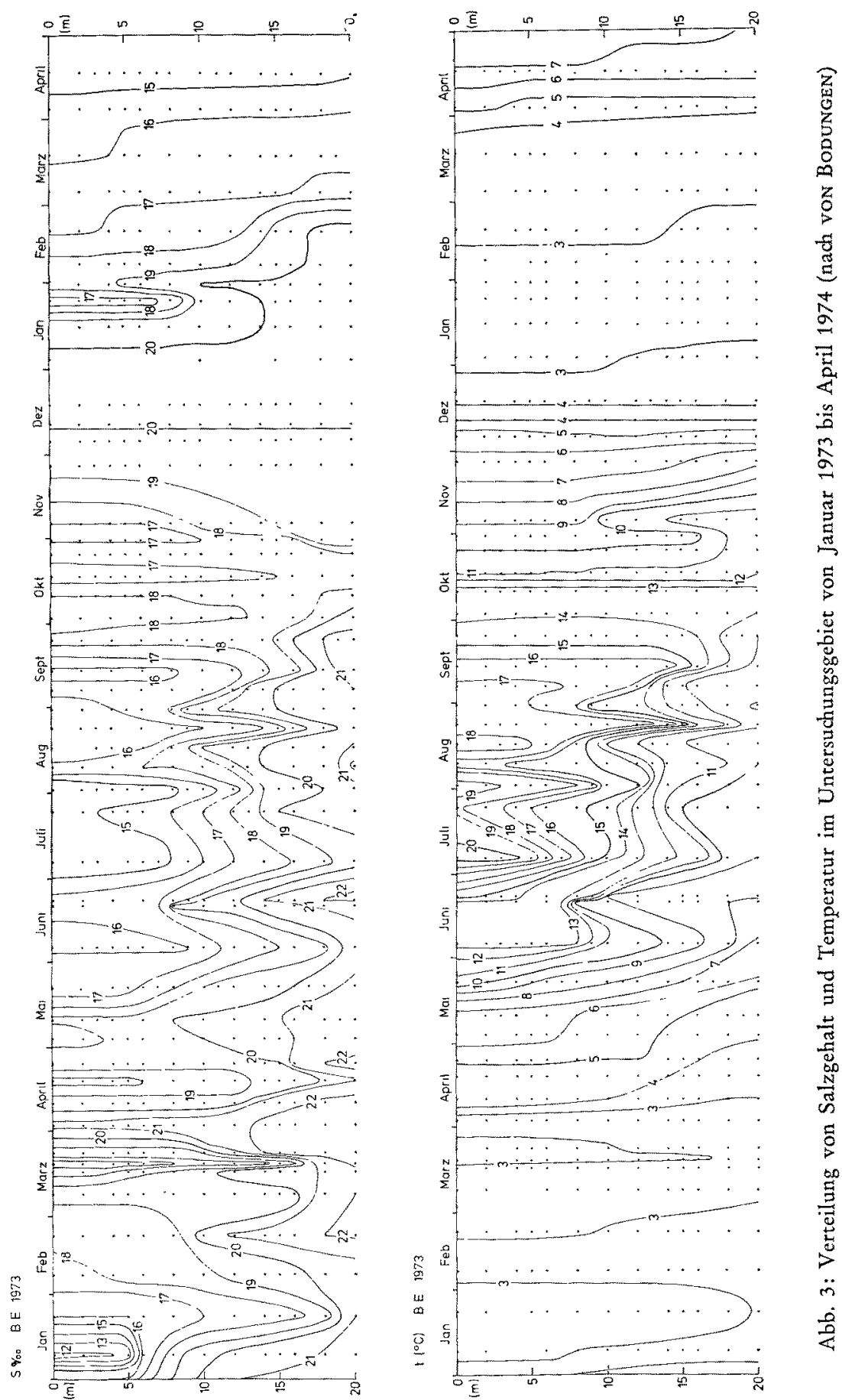


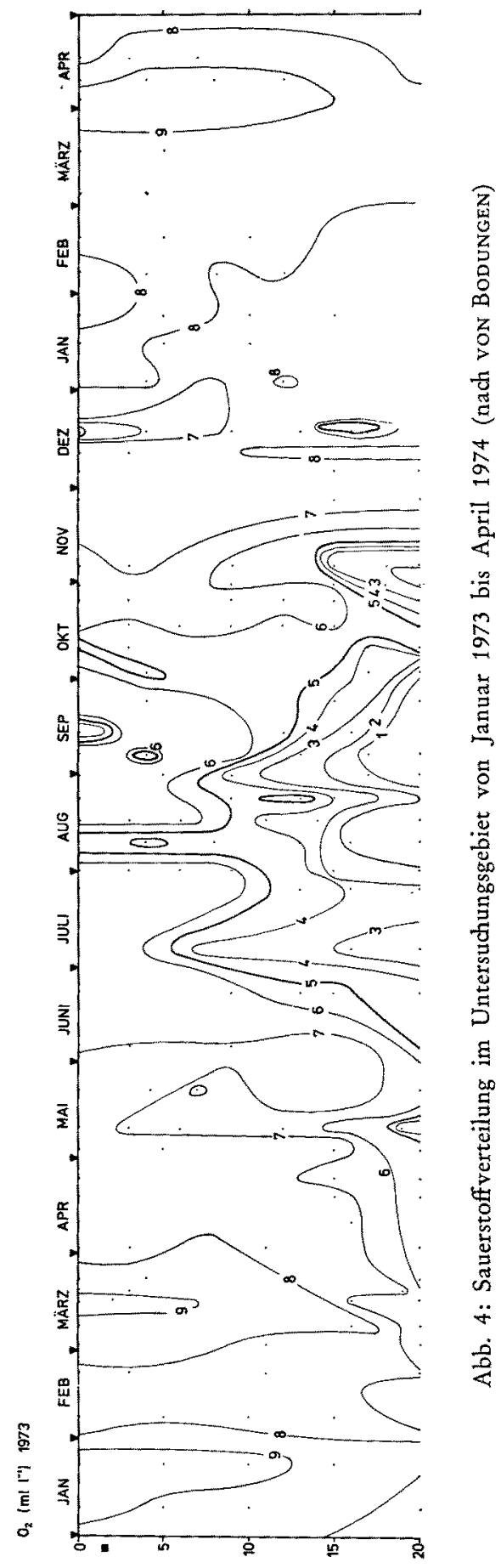


der etwa von $6 \mathrm{~m}-22 \mathrm{~m}$ Tiefe reicht. Die Konsistenz des Sediments ist sehr unterschiedlich. Eine detaillierte Beschreibung der Sedimentverhältnisse im Gebiet des „Hausgartens" wurde von WEFER und Tauchgruppe Kiel (1974) gegeben (Abb. 2). Hiernach liegen die Medianwerte des Sediments bis $12,5 \mathrm{~m}$ Tiefe bei $2,0-0,63 \mathrm{~mm}$; von $12,5-17 \mathrm{~m}$ Tiefe bei $0,4-0,31 \mathrm{~mm}$; von $17-23 \mathrm{~m}$ Tiefe bei $0,4-0,08 \mathrm{~mm}$ und tiefer bei 0,1 bis $0,04 \mathrm{~mm}$. Diese unterschiedlichen Sedimentqualitäten beeinflussen entscheidend die $\mathrm{Zu}$ sammensetzung der Meiofauna.

\section{PHYSIKALISCHE FAKTOREN}

Außer der Sedimentverteilung werden als weitere anorganische Faktoren die Temperatur, Salzgehalt und Sauerstoffverteilung für den Zeitraum von Januar 1973 bis April 1974 berücksichtigt, um das Bild der Verhältnisse im Untersuchungsgebiet zu vervollständigen. Die betreffenden Messungen wurden durch voN BoDUNGEN (1975) und die Gruppe Planktologie des SFB 95 durchgeführt. Ort der Messungen ist ein etwa im Zentrum des Sperrgebietes installierter Forschungsturm (Abb. 2). Erfaßt wird ein Tiefenbereich von $0-20 \mathrm{~m}$ (Abb. 3 und 4$)$.

\section{BESIEDLUNGSMUSTER UND BIOMASSE}

Das Besiedlungsmuster ändert sich in charakteristischer Weise mit der Konsistenz des Sedimentes. Im flachen Bereich $(6-8 \mathrm{~m})$ auf sandig-kiesigen Sedimenten ist die Meiofauna sehr heterogen. Alle Gruppen sind mehr oder weniger stark vertreten,

Tabelle 1

Individuendichte und Biomasse im Vergleich auf den vier untersuchten Stationen

\begin{tabular}{|c|c|c|c|c|}
\hline \multicolumn{5}{|c|}{$\varnothing$-Individuendichte $/ 10 \mathrm{~cm}^{2}$} \\
\hline Tiefe $(\mathrm{m})$ & 6-8 & $12-15$ & $20-22$ & 26 \\
\hline Nematoden & 178 & 414 & 1994 & 1078 \\
\hline Harpacticoiden & 89 & 58 & 1 & - \\
\hline Ostracoden & 6 & 11 & - & - \\
\hline Halacariden & 12 & 16 & - & - \\
\hline Tardigraden & 5 & 1 & - & - \\
\hline Gastrotrichen & 10 & 5 & - & - \\
\hline Gesamtindividuenzahl $/ \mathrm{m}^{2}(\varnothing)$ & 300000 & 505000 & 1950000 & 1078000 \\
\hline \multicolumn{5}{|c|}{$\varnothing$-Biomasse $/ 10 \mathrm{~cm}^{2}$ (Trockengewicht in $\mathrm{mg}$ ) } \\
\hline Tiefe (m) & 6-8 & $12-15$ & $20-22$ & 26 \\
\hline Nematoden & 0,045 & 0,154 & 0,560 & 0,308 \\
\hline Harpacticoiden & 0,063 & 0,124 & - & - \\
\hline \multicolumn{5}{|l|}{ Ostracoden } \\
\hline $\begin{array}{l}\text { Halacariden } \\
\text { Tardigraden }\end{array}$ & 0,042 & 0,072 & - & - \\
\hline \multicolumn{5}{|l|}{ Gastrotrichen I } \\
\hline$\varnothing$-Gesamtgewicht $/ \mathrm{m}^{2}$ & 50 & 116 & 560 & 308 \\
\hline
\end{tabular}


wenn auch die Nematoden dominieren. Mit abnehmender Korngröße und gleichzeitig zunehmender Tiefe bestimmen die Nematoden zunehmend das Bild. In $26 \mathrm{~m}$ Tiefe (Schlick) wurden ausschließlich Nematoden gefunden (Abb. 5). Dies steht weitgehend im Einklang mit früheren Ergebnissen von einigen Stationen unterschiedlichen Sedimentes in der Kieler Bucht (SCHEIBEL \& NoOdT, 1975).

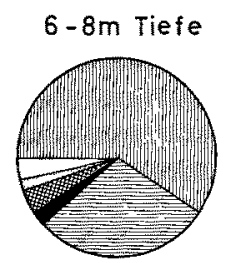

$20-22 m$ Tiefe

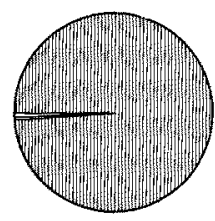

$12-15 \mathrm{~m}$ Tiefe

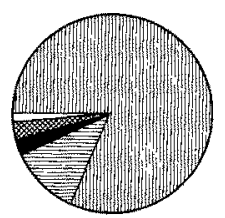

$26 \mathrm{~m}$ Tiefe

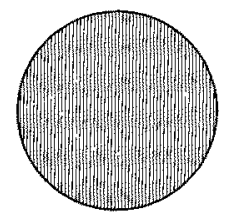
LIIIIII Nematoda

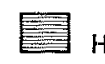

Harpacticoidea Tardigrada
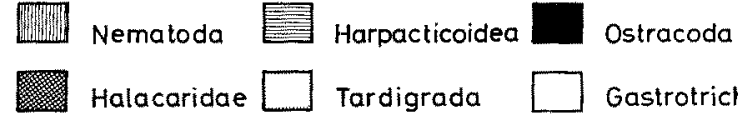
Halacaridae

Gastrotricha

Abb. 5: Prozentuale Verteilung der Meiofauna auf den untersuchten Stationen

Die höchste Individuendichte und Biomasse wird auf den schlickig-feinsandigen Sedimenten und im Schlick erreicht. In diesem Bereich sind es, wie oben erwähnt, allerdings fast ausschließlich Nematoden (Tab. 1).

Ostracoden, Halacariden, Tardigraden und Gastrotrichen werden hinsichtlich ihrer Biomasse zusammengefaßt, da ihre jeweilige Individuenmenge für eine hinreichend exakte Gewichtsbestimmung nicht ausreichte.

\section{MERKMALE DER HARPACTICOIDEN-FAUNA}

In der Kieler Bucht werden durch Harpacticoiden bevorzugt die gröber-sandigen Sedimente besiedelt. Nur in diesem Bereich sind nennenswerte Individuenzahlen zu verzeichnen. Schon auf schlickigem Feinsand ( $20 \mathrm{~m}$ Tiefe) nimmt die Zahl der Tiere sehr stark ab. Entweder enthielten die Probenkerne nur wenige oder einzelne Exemplare, welche allerdings für dieses Sediment charakteristisch sind. $\mathrm{Zu}$ nennen sind hier z. B. Danielssenia typica Bosck, Laophonte brevifurca Sars, (neu für die Kieler Bucht), Laophonte thoracica BoEck. Böden dieser Sedimentqualität sind in einem größeren Areal in der Kieler Bucht von BEcker (1970) nach qualitativen Gesichtspunkten ausführlicher bearbeitet worden. 
Im flacheren Bereich (ca. 6-15 m Tiefe) zeigt die Harpacticoiden-Fauna typische Formen, die mehr oder weniger stark an reine Sande gebunden sind. Entsprechend dem weitreichenden Spektrum der Korngrößen ist auch die Präsenz sandtypischer Arten; im gesamten Tiefenbereich sind sowohl Arten des feineren, homogenen Sandes als auch gröberen Sandes vertreten. Es überwiegen Arten, die gröberen Sand (Medianwert ca. 0,6-1,0 mm) bevorzugen, wie z. B. Amphiascoides debilis GIEsbrecht, Paramphiascopsis longirostris (Claus), Amphiascus minutus (Claus) sp. I LANG, Intermedopsyllus intermedius T. \& A. Scotт, Mesochra inconspicua (T. ScotT), Asellopsis intermedia T. ScotT und Pilifera gracilis (T. ScotT). Arten wie Hastigerella tenuissima (KLIE), Evansula pygmaea (T'. ScotT) und Kliopsyllus bolsaticus Kunz, die für feineren,

Tabelle 2

Abundanz der gefundenen Harpacticoidenarten auf den vier untersuchten Stationen

\begin{tabular}{|c|c|c|c|c|}
\hline Tiefe $(\mathrm{m})$ & $6-8$ & $12-15$ & $20-22$ & 26 \\
\hline Hastigerella tenuissima & 5 & 11 & - & - \\
\hline Halectinosoma gotbiceps & - & 1 & - & - \\
\hline Danielssenia typica & 1 & 1 & 10 & - \\
\hline Tisbe spec. & - & 1 & - & - \\
\hline Dactylopodia vulgaris & 11 & 12 & - & - \\
\hline Paradactylopodia latipes & - & 2 & - & - \\
\hline Amphiascoides debilis & 85 & 33 & 1 & - \\
\hline Paramphiascopsis longirostris & 49 & 104 & 1 & - \\
\hline Paramphiascopsis giesbrechti & - & 4 & - & - \\
\hline Amphiascus minutus sp. I & 65 & 85 & - & - \\
\hline Paramphiascella spec. & - & 3 & - & - \\
\hline Schizopera spec. & - & 1 & - & - \\
\hline Proameira biddensöensis & 1 & - & - & - \\
\hline Ameira parvula & 11 & 4 & - & - \\
\hline Ameira divagans & 3 & 4 & - & - \\
\hline Nitocra spec. & - & 9 & - & - \\
\hline Leptomesochra macintosbi & 4 & - & - & - \\
\hline Scottopsyllus minor & 6 & 21 & - & - \\
\hline Intermedopsyllus intermedius & 52 & 101 & - & 一 \\
\hline Kliopsyllus bolsaticus & 10 & 5 & - & - \\
\hline Mesocbra inconspicua & 22 & 29 & - & - \\
\hline Mesochra pygmaea & 1 & 3 & - & - \\
\hline Leptastacus laticaudatus interm. & 18 & 17 & - & - \\
\hline Leptastacus macronyx & 13 & 41 & - & - \\
\hline Leptopontia curvicauda & 2 & 14 & - & - \\
\hline Paraleptastacus bolsaticus & 2 & 2 & - & - \\
\hline Evansula pygmaea & - & 3 & - & - \\
\hline Evansula incerta & - & 1 & - & - \\
\hline Huntemannia jadensis & 2 & - & - & - \\
\hline Rbizotbrix minuta & 61 & 16 & - & - \\
\hline Heterolaophonte minuta & 3 & 8 & - & - \\
\hline Paronychocamptus curticaudatus & 4 & 6 & - & - \\
\hline Asellopsis intermedia & 13 & 21 & - & - \\
\hline Pilifera gracilis & 16 & 1 & - & - \\
\hline Laophonte brevifurca* & 10 & 17 & 1 & - \\
\hline Laophonte thoracica & - & 1 & 1 & - \\
\hline Onychocamptus brevispinosus" & - & 1 & - & - \\
\hline neu für die Kieler Bucht & & & & \\
\hline
\end{tabular}


homogenen Sand (Medianwert ca. 0,2-0,3 mm) typisch sind, treten in nur kleinen Individuenzahlen auf. Kliopsyllus bolsaticus Kunz kann ein sandiges Sediment in der Kieler Bucht regelrecht charakterisieren (SCHEIBEL \& NoODT, 1975). Ahnliches gilt auch für Nematoden (z. B. Wreser, 1960; Hopper \& MEYers, 1967).

Abschließend kann gesagt werden, daß auf dem untersuchten Profil die Indivi-
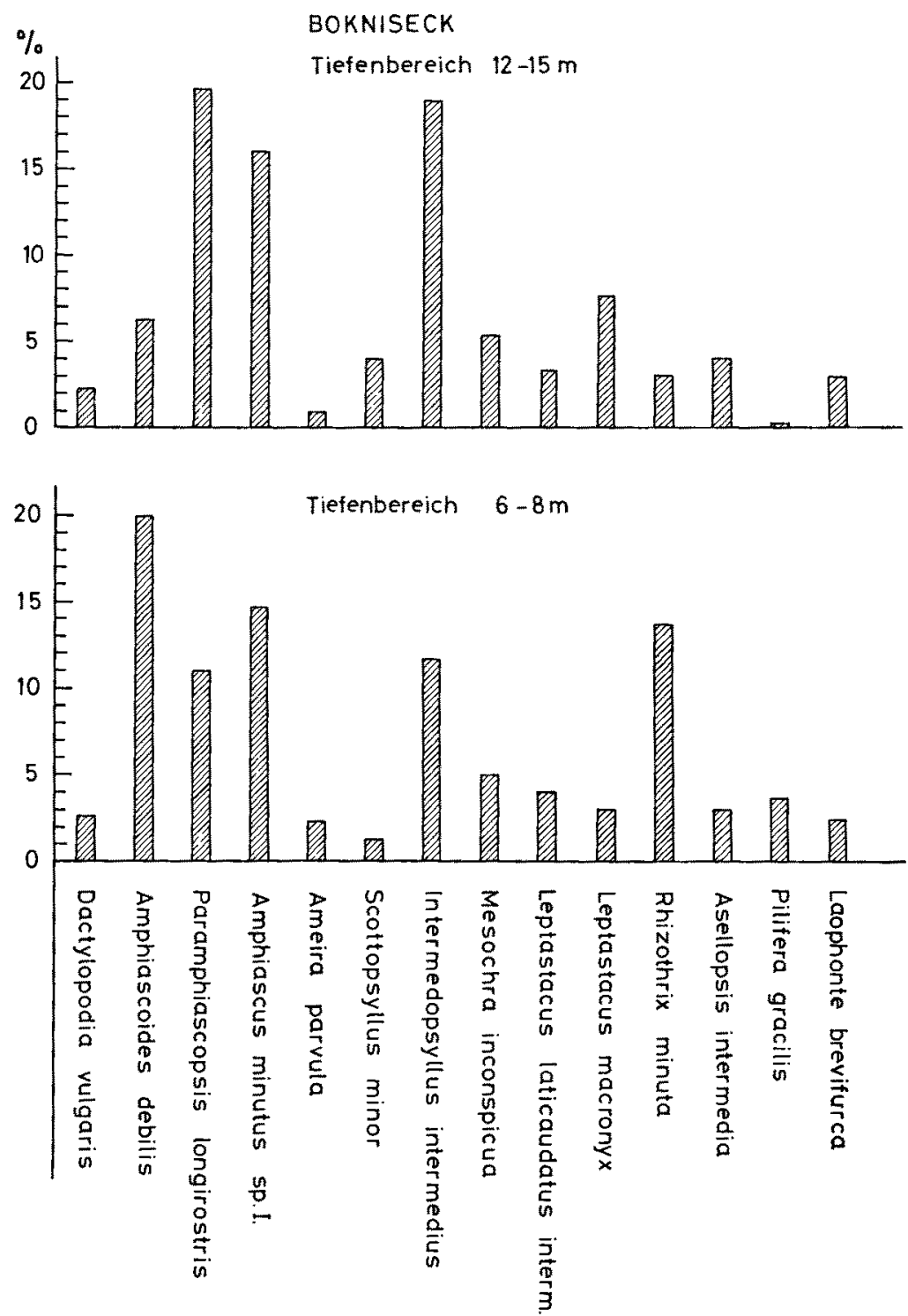

Abb. 6: Prozentualer Anteil der häufigsten Harpacticoidenarten auf den Stationen mit sandigem Sediment 
duenzahl der Harpacticoiden mit abnehmender Korngröße ebenfalls stark zurückgeht. Abbildung 6 zeigt die Abundanz der häufigsten Arten.

Tabelle 2 gibt die gefundenen Arten und ihre Individuenzahlen auf den unterschiedlichen Sedimenten an.

\section{OSTRACODEN, HALACARIDEN, TARDIGRADEN, GASTROTRICHEN}

Individuen dieser Gruppen treten fast ausschließlich im Bereich zwischen 6 und $15 \mathrm{~m}$ auf. Am relativ häufigsten sind Ostracoden und Halacariden. Die Zahlen beider bleiben aber immer erheblich unter der der Harpacticoiden und in noch viel stärkerem Maße unter der der Nematoden. Tardigraden und Gastrotrichen waren meist nur in geringer Zahl in den Probenkernen enthalten oder fehlten gänzlich. Dies ist sicher auf die Heterogenität des Sediments zurückzuführen. Aus praktischen Gründen wurden deshalb diese Gruppen bei der Bestimmung der Biomasse zusammengefaßt. Im Gegensatz zur westlichen Ostsee können in ihrem nördlichen Teil z. B. Ostracoden eine weitaus größere Rolle spielen; bis zu 50\% der Biomasse der Meiofauna (untersucht von Ankar \& Elmgren, 1975). In $26 \mathrm{~m}$ Tiefe (Mudd) fehlen Vertreter dieser Gruppen gänzlich.

\section{DISKUSSION}

Die Ergebnisse bestätigen im wesentlichen frühere Untersuchungen auf unterschiedlichen Sedimenten in der Kieler Bucht (Scherbel, 1973; Scheibel \& Noodt, 1975). Das gilt für die Meiofauna in qualitativer und quantitativer Hinsicht. So zeigen reine Sande je nach Sortierungsgrad ein spezifisches Artenmuster der Harpacticoiden. Mit der Zunahme der Heterogenität des Sedimentes steigt die Artenzahl. Sie nimmt im homogenen Sediment ab und kann durch ein oder wenige Faunenelemente ein Sediment charakterisieren. Auch für die Nematoden-Fauna gilt in der Kieler Bucht eine spezifische Artenverteilung auf sandigen Zonen gegenüber schlickig-sandigen Gebieten (Gerlach, 1958). Warwick \& Buchanan (1970) geben an, daß die Heterogenität der Nematoden-Fauna im sandigen Sediment höher als im schlickigen ist und die Wassertiefe für die Artenzusammensetzung von geringerer Bedeutung als der Sedimenttyp. Das gilt sicher auch für die Kieler Bucht, denn hier können Sandzonen tiefer reichen als Zonen mit schlickigem Charakter und umgekehrt. Eine Analyse der Nematoden des vorliegenden Materials steht noch aus.

Für die Harpacticoiden-Fauna im Untersuchungsgebiet bleibt der Sedimenttyp weitgehend bestimmend; Temperatur und Salzgehalt haben einen geringeren Einfluß. Ebenso wird auch die Komposition der verschiedenen Meiofauna-Gruppen vom jeweiligen Sedimenttyp ganz wesentlich bedingt. In gröberen reinen Sanden dominieren in der Regel die Harpacticoiden (z. B. Coull, 1969; TIETJen, 1969; Scheibel, 1973). Hier muß allerdings berücksichtigt werden, daß die vertikale Verbreitung der Nematoden im Boden gegenüber anderen Meiofauna-Gruppen größer ist und somit ihre volle Zahl nicht exfaßt wird.

Die im Sublitoral der Kieler Bucht erreichte Individuendichte ist mit derjenigen anderer, insbesondere auch vollmariner Meeresgebiete ähnlicher Tiefe, durchaus ver- 
gleichbar (z. B. Coull, 1969; Tietjen, 1969; Stripp, 1969; McIntyre, 1964; Wigley \& MCINTYRE, 1964; WIESER, 1960), wenn hier natürlich auch die unterschiedlichen Bedingungen der jeweiligen Lokalitäten mehr oder weniger starken Einfluß ausüben.

Auch die Werte der Biomasse liegen im wesentlichen im Bereich derer, die von anderen Autoren ermittelt wurden. Höhere Werte wurden allerdings von STRIPP (1969) in einem Areal der Nordsee errechnet. Gewisse Differenzen sind in dieser Hinsicht nicht auszuschließen, da von verschiedenen Autoren unterschiedliche Methoden zur Ermittlung der Biomasse angewandt wurden. Stripp (1969), McIntyre (1973), ANKar \& Elmgren (1975) und TIETJEn (1969) kalkulieren die Gewichte; andere Autoren wägen direkt und geben die Werte als Trockengewicht an (z.B. Cout, 1969; WARWICK \& Buchanan, 1971).

Im Untersuchungsgebiet treten die höchsten Werte der Biomasse im schlicksandigen Bereich (ca. 18-22 m) und im Schlick auf (26 m). Hier sind es fast ausschließlich Nematoden, die sie ausmachen. Eine ähnlich große Rolle spielen die Nematoden auch im brackigeren Bereich der Ostsee. Biomasse und Individuenzahl können hier aber höher sein (z. B. Elmgren, 1975; ANKar \& Elmgren, 1975). Im Durchschnitt geringere Individuenzahlen werden von ARLT (1973) angegeben. Sediment und Tiefe sind allerdings nur bedingt direkt vergleichbar.

Allgemeinere Probleme, die Vorkommen und Zusammensetzung der Meiofauna betreffen, sollen an dieser Stelle nicht erörtert werden. Hierüber ist von vielen Autoren in jüngster Zeit berichtet worden (z. B. MCINTYRE, 1973).

\section{ZUSAMMENFASSUNG}

1. In einem Versuchsgebiet der westlichen Kieler Bucht wurden über einen Zeitraum von mehreren Monaten quantitative Proben durch Taucher entnommen. Jeder Probenkern erfaßt eine Fläche von $10 \mathrm{~cm}^{2} \cong 50 \mathrm{~cm}^{3}$. Die Untersuchungen erfolgten auf einem Profil unterschiedlicher Sedimentqualitäten (Grobsand bis Schlick) an festen Stationen.

2. Jeder Sedimenttyp zeigt eine spezifische Besiedlung durch Meiofauna, speziell durch Harpacticoiden und Nematoden. Die durchschnittliche Individuendichte $/ \mathrm{m}^{2}$ betrug 300 000-505 000 Individuen auf mittlerem bis grobem Sand, 1950000 Individuen auf schlickigem Feinsand und 1078000 Individuen auf Schlick.

3. Die durchschnittliche Biomasse/ $\mathrm{m}^{2}$ betrug $50-116 \mathrm{mg}$ auf mittlerem bis grobem Sand, $560 \mathrm{mg}$ auf schlickigem Feinsand und $308 \mathrm{mg}$ auf Schlick.

4. Diese Ergebnisse werden mit denen aus anderen Meeresgebieten verglichen. Dabei zeigt sich, daß sie im wesentlichen in der gleichen Größenordnung liegen.

Danksagung. Der Tauchgruppe der Universität Kiel möchte ich an dieser Stelle für ihren Einsatz bei der Probenentahme besonders danken.

\section{ZITIERTE LITERATUR}

ANkar, S. \& Elmgren, R., 1975. A survey of the benthic macro- and meiofauna of the AsköLandsort area. Merenstutkimuslait. Julk 239, 257-264. 
ArLT, G, 1973a. Vertical and horizontal microdistribution of the meiofauna in the Greifswalder Bodden. Oikos (Suppl.) 15, 105-111

- 1973. Jahreszeitliche Fluktuationen der Meiofauna im Greifswalder Bodden. Wiss. Z. Univ. Rostock 22, 685-692.

Ax, R., 1951. Die Turbellarien des Eulitorals der Kieler Bucht. Zool. Jb. (Syst. Okol. Geogr. Tiere) 80, 277-378.

- 1952. Turbellarien der Gattung Promesostoma von den deutschen Küsten. Kieler Meeresforsch. 8, 218-226.

BeCKer, K. H., 1970. Beitrag zur Kenntnis der Copepoda Harpacticoidea sublitoraler Weichböden in der Kieler Bucht. Kieler Meeresforsch. 26, 56-73.

Bodungen, B. von, 1975. Der Jahresgang der Nährsalze und der Primärproduktion des Planktons in der Kieler Bucht unter Berücksichtigung der Hydrographie. Diss. Kiel.

Coutl, B. C., 1969. Shallow water meiobenthos of the Bermuda platform. Oecologia 4, 325-357.

ELMGREN, R., 1975. Benthic meiofauna as indicator of oxygen conditions in the Northern Baltic proper. Merentutkimuslait. Julk 239, 265-271.

GerLaCH, S. A., 1958. Die Nematodenfauna der sublitoralen Region in der Kieler Bucht. Kieler Meeresforsch. 14, 64-90.

Hartmann, G., 1956. Neue Funde von Muschelkrebsen (Ostracoda) in Gebiet der Nordseeküste und der Kieler Budht (mit Beschreibung einer neuen Art). Schr. naturw. Ver. Schlesw.Holst. 29, 103-111.

Hopper, B. E. \& Meyers, S. P., 1967. Population studies on benthic nematodes within a subtropical seagrass community. Mar. Biol. 1, 85-96.

KLIE, W., 1929. Die Copepoda Harpacticoidea der südlichen und westlichen Ostsee mit besonderer Berücksichtigung der Sandfauna der Kieler Bucht. Zool. Jb. (System Okol., Geogr. Tiere) 57, 329-386.

- 1950. Harpacticoiden (Cop.) aus dem Bereich von Helgoland und der Kieler Bucht. II. Kieler Meeresforsch. 7, 76-128.

Kunz, H., 1935. Zur Okologie der Copepoden Schleswig-Holsteins und der Kieler Bucht. Schr. naturw. Ver. Schlesw.-Holst. 21, 84-132.

LANG, K., 1948. Monographie der Harpacticiden. Lund, 1-2.

MCINTYRE, A. D., 1964. Meiobenthos of sublitoral muds. J. mar. biol. Ass. U.K. 44, 665-674.

- 1973. The meiofauna of a flatfish nursery ground. J. mar. biol. Ass. U.K. 53, 93-118.

Remane, A., 1933. Verteilung und Organisation der benthonischen Mikrofauna der Kieler Bucht. Wiss. Meeresunters. 21, 161-221.

Scheibez, W., 1973. Quantitativ-ökologische Untersuchungen am uferfernen Mesopsammon in der Kieler Bucht. Kieler Meeresforsch. 29, 58-68.

- \& Noodt, W., 1975. Population densities and characteristics of meiobenthos in different substrates in the Kiel Bay. Merentutkimuslait. Julk 239, 173-178.

Stripp, K., 1969. Das Verhältnis von Makrofauna und Meiofauna in den Sedimenten der Helgoländer Bucht. Veröff. Inst. Meeresforsch. Bremerh. 12, 143-148.

TiETJEn, J. H., 1969. The ecology of shallow water meiobenthos of two New England estuaries. Oecologia 2, 251-291.

WARWICK, R. M. \& BUChaNAN, J. B., 1970. The meiofauna off the coast of Northumberland. I. The structure of the nematode population. J. mar. biol. Ass. U.K. 50, 129-146.

Wefer, G., 1974. Topographie und Sedimente im „Hausgarten“ des Sonderforschungsbereiches 95 der Universität Kiel (Eckernförder Bucht, Westl. Ostsee). Meyniana 26, 3-7.

Wieser, W., 1960. Benthic studies in Buzzards Bay. II. The meiofauna. Limnol. Oceanogr. 5, 121-137.

WIGLEY, R. L. \& MCINTYRE, A. D., 1964. Some quantitative comparisons of off shore meiobenthos and macrobenthos south af Martha's Vineyard. Limnol. Oceanogr. 9, 485-493.

Anschrift des Autors: Dr. W. Scheibel

Zoologisches Institut der Universität Kiel

Hegewischstr. 3

D-2300 Kiel

Bundesrepublik Deutschland 